\section{Derechos reproductivos: autonomía y objeción de conciencia en el sur de Chile*}

Reproductive rights: autonomy and conscientious objection in the southern Chile

\author{
YANIRA ZUÑIGA A.* \\ DebBie GuerRa M.* \\ Susana Rebolledo B. ${ }^{* * * *}$
}

\section{Resumen}

El artículo analiza las nociones de autonomía y de vida subyacentes en los discursos de profesionales de salud sexual y reproductiva

\footnotetext{
Proyecto FONDECYT No 1150796. "Los Derechos Sexuales y Reproductivos. Entre el Control y la Autonomía".

** Instituto de Derecho Público. Facultad de Ciencias Jurídicas y Sociales, Universidad Austral de Chile. E mail: yzuniga@uach.cl

*** Instituto de Estudios Antropológicos, Facultad de Filosofía y Humanidades, Universidad Austral de Chile. E mail: dguerra@uach.cl

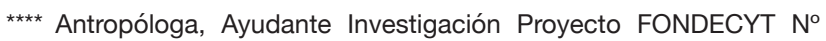
1150796. E-mail: susi.rebo@gmail.com
}

en la ciudad de Valdivia. Para ello, se realiza un estudio de caso cualitativo que analiza los temas del valor de la vida emergente, los principios bioéticos, los derechos sexuales y reproductivos y la objeción de conciencia. Los resultados indican que los avances normativos respecto de la ciudadanía biológica se ven limitados por el saber médico y los valores de las y los profesionales de salud sexual y reproductiva.

Palabras clave: Derechos sexuales y reproductivos, principios bioéticos, autonomía, objeción de conciencia.

\begin{abstract}
The article analyzes the underlying concepts of life and autonomy of the discourses of sexual and reproductive health professionals in the city of Valdivia. Through a qualitative case study, it addresses the values given to emerging life, bioethical principles, sexual and reproductive rights, and conscientious objection. Results show that the medical knowledge and the normative values of the sexual and reproductive health professionals constrain the exercise of biological citizenship.
\end{abstract}

Key words: Sexual and reproductive rights, bioethical principles, autonomy, conscientious objection. 


\section{Introducción}

La relación clínica, es decir, los vínculos entre pacientes y personal de salud, ha experimentado notables transformaciones en las últimas décadas. El hospital ha sido permeado por el paulatino ensanchamiento de la idea de ciudadanía. Aspectos de la vida humana que eran entendidos como parte de un destino o regularidad biológica, han empezado a concebirse como esferas de deliberación y decisión individuales, abriendo paso a la ciudadanía biológica o en el hospital (Novas y Rose 2003: 458).

Sin embargo, la ciudadanía en el hospital es altamente problemática. Como ha puesto de relieve Foucault (1993, 2003, 2010), la misma razón ilustrada en la que se enraizó la autonomía individual y la dignidad sirvió de asiento a un nuevo poder - el biopoder - dirigido a vigilar y controlar las conductas humanas, a través de los cuerpos individuales.

De ahí que el hospital haya devenido un territorio disputado por dos fuerzas. Una, vinculada al poder-saber de la medicina que reclama para sí el monopolio de las decisiones sobre los cuerpos de los pacientes (Lemm 2010: 17); y otra que reivindica la capacidad moral de cada individuo para adoptar decisiones referentes a su propia salud y vida, sin interferencia ajena.

Desde el siglo XIX en adelante, el modelo paternalista-caracterizado por la obediencia/ pasividad/dependencia del paciente y por la autoridad incontestada de la decisión médicaha predominado en la relación clínica. En las últimas décadas, las normas jurídicas han intentado democratizar dicho vínculo. La hegemonía del poder-saber médico ha debido ceder terreno ante la tendencia expansiva de normas de derechos humanos y el desarrollo de los estatutos de responsabilidad médica. Así, por ejemplo, la ley $\mathrm{N}^{\circ} 20.584$ (2012) que regula los derechos y deberes que tienen las personas en relación con acciones vinculadas a su atención en salud contempla el derecho al trato digno (art. 5), el derecho a la información (artículos 8 al 11), y el derecho a otorgar o denegar la voluntad para someterse a cualquier procedimiento o tratamiento de salud (art. 14). Correlativamente, impone a los prestadores de salud obligaciones de confidencialidad en relación con la ficha clínica de cada paciente (arts. 12 y 13), deberes de adecuación de lenguaje (art. 5.a), respeto de reglas de cortesía (art. 5.b), y obligaciones de suministro de información comprensible e integral (art. 8), entre otras disposiciones.

A pesar de estos desarrollos jurídicos y del creciente protagonismo de las organizaciones de pacientes en los debates sobre política pública sanitaria, la ciudadanía en el hospital es un ideal normativo que enfrenta diversos obstáculos para su consolidación. En general, la disposición anímica de los y las pacientes frente al personal de salud continúa siendo más cercana a la actitud de quien espera un acto de buena voluntad, que a la de quien ejerce un derecho o potestad jurídica (Solitario, et al, 2008). Por otra parte, el desarrollo exponencial de la objeción de conciencia, particularmente en el marco de la salud reproductiva, impone severas restricciones a la autonomía de las y los pacientes y afecta la garantía de los derechos a la vida y a la salud (Casas y Dides, 2007; Montero, 2014; Alegre, 2010).

En la medida de que las decisiones sobre la sexualidad y la reproducción femeninas han sido históricamente tuteladas, y que la producción 
de discursos científico-médicos ha servido a estabilizar el orden social de género (Foucault, 1993; Boyer 2012; Llober-Valls, 2009) el examen de la relación clínica en el marco de la salud reproductiva ofrece un inmejorable caso de estudio para observar los cambios, resistencias y obstáculos en el establecimiento de una ciudadanía biológica respecto de colectivos discriminados.

Este trabajo examina los discursos de las y los profesionales vinculados a la atención de salud reproductiva sobre temas tales como los derechos sexuales y reproductivos, la vida, la autonomía sexual y reproductiva, y la objeción de conciencia; intenta trazar las nociones de autonomía y de vida que subyacen a estos discursos y ofrece una reflexión sobre los desafíos socio-normativos que involucran estos hallazgos.

\section{Metodología}

A objeto de conocer el discurso del personal de salud en torno al valor de la vida emergente, la autonomía sexual y reproductiva, el derecho a la salud y la objeción de conciencia, se realiza un estudio cualitativo que permite "comprender las formas en las que el mundo social es interpretado, comprendido, experimentado y producido [...] se basa en la generación de datos flexibles y sensibles al contexto social en el que se producen" (Vasilachis 2006: 25).

El método utilizado es el de estudio de casos instrumental que según Stake (2005) es un estudio en profundidad que busca comprender de modo general $y$, a partir de un conjunto de situaciones específicas, una situación paradójica.
La técnica de recolección de información consiste en una entrevista semi-estructurada, entendida como "una comunicación interpersonal establecida entre el investigador y el sujeto de estudio, a fin de obtener respuestas verbales a las interrogantes planteadas sobre el problema propuesto" (Canales 2006: 163-165). Este tipo de entrevista se adapta a las y los participantes del estudio, permitiendo aclarar términos, identificar ambigüedades y temas emergentes.

La pauta de entrevista semi-estructurada está compuesta de 5 ítems y 29 preguntas formuladas a partir del objetivo del proyecto y del marco teórico, a saber: Derecho a la salud, autonomía y control sexual y reproductivo, objeción de conciencia, aborto y valor de la vida. Las entrevistas son grabadas con el consentimiento de cada uno y una de las participantes del estudio y tienen una duración entre 60 a 90 minutos aproximadamente. Las grabaciones son transcritas en su totalidad. Las respuestas son sometidas a análisis de contenido, es decir, una técnica de carácter interpretativo que a partir de ciertos datos genera inferencias reproducibles y válidas que puedan aplicarse al contexto donde tienen lugar los mensajes y sus significados (Krippendorff 1990). La información es codificada en base a categorías de análisis derivadas de los objetivos y marco teórico.

Esta entrevista es aplicada antes de la entrada en vigencia de la Ley Núm. 21.030 que Regula la Despenalización de la Interrupción Voluntaria del Embarazo en Tres Causales (Ley Núm. 21.030 de aquí en adelante) y la Norma Técnica Nacional de Acompañamiento y Atención Integral a la Mujer que se Encuentra en Alguna de las Tres Causales que Regula la Ley Núm. 21.030 (Norma Técnica de Acompañamiento y 
Atención Tres Causales de aquí en adelante). Cabe mencionar que antes de la entrada en vigencia esta normativa, no existía ninguna disposición jurídica que regulara, de manera explícita, la objeción de conciencia.

Los criterios de rigor considerados son la credibilidad o criterio de verdad, esto se refiere al grado en que los resultados dan cuenta de una realidad o situación dada. En este caso, el criterio de credibilidad se asegura a través de la transcripción fidedigna de las entrevistas y la triangulación de investigadoras. La confirmabilidad o auditabilidad del estudio se basa en la descripción de las y los participantes, uso de grabaciones y transcripciones fieles a sus dichos.

Las y los participantes son profesionales del área de salud gineco-obstetra, a saber, matronas

Tabla 1: Datos Profesionales Consultados.

\begin{tabular}{|l|c|l|}
\hline \multicolumn{1}{|c|}{ PROFESIÓN } & AÑOS DE SERVICIO & SECTOR DESEMPEÑO \\
\hline Obstetricia y Puericultura & 20 & Público \\
\hline Obstetricia y Puericultura & 20 & Público \\
\hline Obstetricia y Puericultura & 5 & Público \\
\hline Obstetricia y Puericultura & 5 & Público \\
\hline Obstetricia y Puericultura & 3 & Público \\
\hline Obstetricia y Puericultura & 3 & Público \\
\hline Ginecología & 28 & Público/Privado \\
\hline Ginecología & 16 & Público/Privado \\
\hline Ginecología & 41 & Público/Privado/ Académico \\
\hline Ginecología & 34 & Público/Privado/Académico \\
\hline Ginecología & 10 & Público/Privado/Académico \\
\hline Obstetricia y Puericultura & 20 & Público/Académico \\
\hline Obstetricia y Puericultura & 20 & Público/Académico \\
\hline Obstetricia y Puericultura & 20 & Público/Académico \\
\hline Obstetricia y Puericultura & 5 & Público/Académico \\
\hline Obstetricia y Puericultura & 3 & Público/Académico \\
\hline Obstetricia y Puericultura & 20 & Privado \\
\hline Obstetricia y Puericultura & 2 & Académico \\
\hline
\end{tabular}

Elaboración propia. 
y matrones y ginecólogas y ginecólogos del sistema de salud público, privado y académico de Valdivia, región de Los Ríos. La muestra es no probabilística, es decir, el muestreo se realiza tanto por conveniencia, o sea se selecciona una muestra de la población por el hecho de que sea accesible, como a través de la técnica bola de nieve; técnica en la que las y los participantes mencionan a nuevos participantes entre sus conocidos. Este tipo de muestra no es representativa $y$, por lo tanto, no permite los procesos de generalización. En ese sentido, las y los participantes son seleccionados al azar a partir de un catastro de profesionales de la ciudad de Valdivia y alrededores y por bola de nieve.

La muestra corresponde a 18 profesionales de salud, a saber, 12 matronas y 1 matrón; 3 ginecólogas y 2 ginecólogos ${ }^{1}$, quienes se contactan a través de correo electrónico y, en algunos casos, vía teléfono celular. Algunas de las personas son entrevistadas en sus lugares de trabajo y otras - a petición de ellas- en un lugar externo. El número de entrevistas realizadas permite cumplir con el criterio de saturación de la información, es decir, cuando "la información recogida resulta sobrada en relación con los objetivos de investigación. Esto es, nuevas entrevistas no añaden nada relevante a lo conocido" (Vallés 2009: 68).

A todas y todos los participantes se les entrega un consentimiento informado indicando el objetivo del estudio, el destino de la información y asegurando su privacidad, anonimato y resguardo de identidad, por ello, se utilizará un código que indica el número de la entrevista y

Entre abril 2017 y enero 2018, se contacta 64 profesionales -33 matronas y matrones y 31 ginecólogas y ginecólogos- de Valdivia y alrededores. Solo los 18 profesionales mencionados responden a la solicitud de contacto. profesión ${ }^{2}$. Asimismo, es necesario mencionar, que la grabadora se apaga cada vez que es solicitado.

\section{Resultados}

Los discursos de las y los profesionales vinculados a la atención de salud reproductiva sobre los temas que nos ocupan en este artículo se entreveran en concepciones de salud y enfermedad "cargados de connotaciones valóricas" (Lolas y Outomuro 2006: 104) construidos social e históricamente.

En las últimas décadas del siglo $X X$ y principios del siglo XXI, la sexualidad y la reproducción emergen como ámbitos de preocupación de las ciencias sociales, medicina y la política, así como menciona Petchesky (2009: 9) "'el sexo es siempre algo político' y su politización involucra establecerlo en los límites entre lo 'bueno y lo malo' basado en una jerarquía de valor sexual en la religión, en la medicina, en las políticas públicas y la cultura popular".

En ese contexto, surge la noción de derechos sexuales y reproductivos asociados al ejercicio de la ciudadanía biológica vinculada a los movimientos de mujeres y de diversidad sexual. Ese ejercicio busca "refundar la relación entre lo público y privado" (Miles 2016: 11), enfatizando en la libertad de todas las personas de decidir sobre aspectos asociados a su sexualidad y reproducción sin coerciones ni violencia.

Con la finalidad de resguardar la identidad de las y los profesionales que participaron en el presente estudio, se realiza la sustitución de sus nombres por un código que contempla $\mathrm{E}=$ entrevista, Número $=$ indica el orden de entrevista y Profesión $=\mathrm{M}$ matrón/a y G ginecóloga/ginecólogo según corresponda. 


\section{Derechos sexuales y reproductivos. Concepto y contenidos}

El análisis de la información en relación a los derechos sexuales y reproductivos se desagrega en dos aspectos: los derechos reproductivos, por un lado y los derechos sexuales, por el otro. Para la gran mayoría de las y los participantes, los derechos reproductivos consisten en una libertad individual (habitualmente de cada mujer) de decidir, sin interferencia ajena, si se desea tener hijos, su número e intervalo. Comprenden ciertos elementos consustanciales para su ejercicio, como la posibilidad de usar métodos anticonceptivos y contar con información relevante:

(...) respecto a los derechos reproductivos, se puede decir que son los derechos de cada persona de poder decidir en qué momento nos vamos a reproducir, con qué espacio, con qué espaciación vamos a tener hijos, si nos reproducimos o no, con quién, cuándo y recibir toda la información necesaria para realizar el control de natalidad, obviamente regular esto mediante información y acceso a los métodos anticonceptivos (E3M).

Esta manera de conceptualizar los derechos reproductivos guarda similitud con las primeras formulaciones jurídicas internacionales, las que se focalizaron en la autonomía procreativa y en las políticas de anticoncepción (p. ej. Proclamación de la Conferencia Internacional de Derechos Humanos de Teherán, de 1968) y la Convención para la Eliminación de todas las formas de Discriminación contra la Mujer, de 1979). El acceso a la información relevante fue incorporado, en la década de los 90, por el Programa de Acción de la Conferencia sobre Población y Desarrollo (Cairo, 1994) y la Declaración de Pekín (1995). Estos últimos instrumentos internacionales han precisado que los derechos reproductivos incluyen, además, el derecho a acceder a prestaciones de salud reproductiva sin sufrir discriminación, coacciones ni violencia, la promoción de la igualdad de género, y la atención especial de las necesidades de la población adolescente en materia de enseñanza y de servicios de salud reproductiva. Tales dimensiones normativas, vinculadas a los principios de no discriminación y de justicia, no aparecen mencionadas por las y los participantes del estudio, como elementos constitutivos de los derechos reproductivos. Tampoco se menciona la posibilidad de optar por la desmedicalización del embarazo y/o el parto, o de determinar las condiciones de este último (por cesárea, con o sin epidural etc.)

Se menciona, en relación a la práctica profesional, la existencia de vínculos con otros derechos o principios normativos, tales como el derecho a la privacidad y la confidencialidad; el derecho a la vida o el respeto a la dignidad. Sin embargo, es necesario precisar que las y los entrevistados en sus discursos no hacen diferencia entre los principios jurídicos que rigen la atención de salud y los principios bioéticos.

Los significados atribuidos a los derechos sexuales por las y los participantes son variados. Si bien la gran mayoría considera que estos derechos protegen la autonomía sexual, es decir, consisten en la facultad de tener relaciones sexuales libres de coerción; algunas respuestas remiten al placer sexual, a la orientación sexual o a la identidad de género, por ejemplo, una de las entrevistadas sugiere: "cada persona puede ejercer su vida sexual de la manera que quiera, en cuanto a género, frecuencia, gustos, etc." (E9G).

\section{Principios que rigen la atención de salud reproductiva}

De acuerdo con las y los entrevistados, la práctica profesional debe sustentarse en 
un amplio crisol de principios. Este abanico comprende tres de los cuatro principios éticos básicos de la biomedicina, esto es, la autonomía, la beneficencia y la no maleficencia A estos principios bioéticos se suman principios de naturaleza jurídica, tales como el acceso a información completa y veraz, la confidencialidad, el respeto a la dignidad o la no discriminación, la educación, la neutralidad y la oportunidad de la atención. Los principios de autonomía, acceso a información completa y veraz y confidencialidad son los que acumulan más menciones. La prelación o importancia de cada uno de ellos varía entre las y los participantes. Así por ejemplo, una de las entrevistadas señala

(...) primero la confidencialidad absoluta, segundo que la veracidad de los hechos, o sea de la información, lo ideal es que esto sea absolutamente confidencial, personal, ya, y sin ninguna presión, o sea yo cuando veo pacientes, si el paciente decide contarme algo, me lo cuenta y está entre el paciente y yo (E6G)

En otro caso se hace referencia a que:

El principio máximo es el bienestar (...) el beneficio del paciente y dejarle a libre elección, en base a información fidedigna, más actualizada, la mejor que se le pueda entregar y que el paciente elija, bajo su criterio (E3M).

\section{La autonomía reproductiva. Concepto y límites}

La autonomía reproductiva, en todos los casos, es definida como libertad de decidir y escoger, sin coerción externa, como lo expresa una de las participantes del estudio: "es la libre elección, la posibilidad de libre elección y de decisión" (E6G).

Para la mayoría de las y los profesionales entrevistados, la autonomía reproductiva no está condicionada por el sexo o por la orientación sexual de cada paciente. En el caso de menores de edad y en supuestos de discapacidad mental, se reporta la existencia de algunas restricciones jurídicas a respetar por el personal de salud (reglas de autoridad parental e interdicciones) y la existencia de ciertas prácticas contrarias al principio de autonomía:

Bueno (...) nunca han tenido hijos, pero no llegan a esterilizarse ellas, a ellas sí se les violenta su derecho a la autonomía porque la que decide ahí es la mamá, niñas con síndrome de Down, niñas con [pausa] alguna secuela de un accidente vascular o una meningitis, la opinión de ellas no vale (E18M).

Pese a la importancia que las y los participantes del estudio le confieren a la autonomía reproductiva, reconocen que esta tropieza con múltiples obstáculos o límites en la práctica clínica. Uno de ellos se relaciona con la configuración del discurso científico-médico como un discurso totalizador. Foucault (2003) explica que la medicina moderna ha dotado a los procesos biológicos que ocurren al interior del cuerpo humano de una existencia propia, que puede abstraerse de las características identitarias del paciente. Por consiguiente, la verdad clínica es susceptible de ser develada únicamente por quien posee una mirada técnica capaz de ver, aislar e identificar lo que hay de normal y de anormal en un conjunto de cuerpos; y requiere, en contrapartida, la evicción del punto de vista del paciente. En esa verdad reside no solo la capacidad de curar, sino también la capacidad de normar la vida de otros.

Las y los pacientes son el objeto de los discursos científico-normativos. En consecuencia, su participación en los procesos de construcción y aplicación de estos discursos, se vuelve disruptiva: 
La gran parte de las normativas ligadas a la reproducción y la sexualidad se basan en la decisión médica, decisión terapéutica, en cómo orientar este tipo de decisiones a lo que es clínico básicamente, pero (...) no hay apartados que digan cómo entregar información al paciente, o dar la oportunidad que las pacientes decidan o tomen decisiones, sino que está súper estipulado, como protocolo, qué cosas seguir, paso a paso, como; se trata de esta forma; se maneja de esta forma; indico tal y tal medicamento, pero en ningún punto se establece la participación del paciente, de la usuaria en su propia salud (E11M).

La autonomía reproductiva también puede resultar limitada debido a la sustitución de la voluntad de las/los pacientes por la decisión del personal de salud, como refiere una de las entrevistadas:

En los derechos sexuales y reproductivos existen normativas que dicen que se le debe ofrecer a la usuaria, se le debe entregar la autonomía de decisión, se le debe atender propendiendo a su, resguardar integridad física y emocional en el lenguaje y en la forma. ¿Cuál es el problema? que eso es una norma y todas las normas tienen que ser llevadas a la práctica por personas y ahí es donde se puede romper esto que está normado, esto está escrito, pero la persona que atiende es quien le pone el sello personal y ese sello puede romper muchas veces el derecho de estas personas a recibir una atención bajo esas condiciones (E16M).

En suma, el ejercicio de la autonomía se ve obstaculizado por una "cultura médica" que se resguarda en una autoridad dada por el saber científico-médico.

Desde la mirada del sistema hoy en día todavía nosotros estamos en pañales en permitirles a las personas ejercer su derecho, nos cuesta, nos cuesta, nos cuesta como funcionarios de salud, nos cuesta como funcionarios en todo ámbito [pausa] ahora qué es lo que nosotros podemos hacer, ofrecerles alternativas, entonces desde mi mirada nos falta todavía el ser capaces de entregarle el derecho al otro y dejar de ser nosotros quienes decidimos por ellos, porque como sabemos más, creemos que sabemos más (E16M).

Un segundo grupo de límites se refiere a la falta de información adecuada. Para la gran mayoría de las y los participantes, la calidad y la cantidad de la información de la que dispone cada paciente son determinantes en la formación de una voluntad libre y autónoma.

Yo considero que algo importante es la información completa, bajo esa condición creo que una persona podría ejercer completamente la autonomía, también bajo un cuidado específico y completo, que tenga un profesional que la acompañe y que le otorgue el cuidado necesario, que a veces una persona por, no estar inserta en el área de la salud por ejemplo, pueda dárselo por sí misma (E11M).

La calidad de la información remite siempre a la expertise clínica de quien la suministra. La cantidad de información no se vincula a un criterio único, se utilizan adjetivos variados tales como completa, íntegra, necesaria o suficiente. Sólo en un caso se menciona la necesidad de que la información sea transmitida en términos respetuosos a los y las pacientes y comprendida por ellos y ellas:

En el caso de un profesional transmitir la información correcta y clara, ojalá no referirse nunca a menos que la otra persona, digamos, que sea receptora de información tenga mucho nivel educacional, siempre referirme en términos muy sencillos, quizás explicar siempre con las palabras que corresponden, pero siempre bajar la información a la persona que uno le está entregando los datos que sé yo la información de lo que sea, o de la atención que se le va a brindar, o el plan que se va a seguir con esa persona desde que ingresa a un hospital por ejemplo (E14M).

En las sociedades modernas, el conocimiento científico traza los criterios de verdad y falsedad respecto de la sexualidad y la procreación, reemplazando la ars erotica por la scientia sexualis (Foucault, 1993). Transmitido a la población, a través de la educación y la consejería clínica, permite erradicar mitos. Así lo sugiere uno de los entrevistados:

(...) para nosotros es súper importante que la mujer tenga autonomía, pero que a la vez estén informadas digamos de lo que va a ocurrir, o sea hoy en día hay mucha 
información disponible en todas las redes sociales, en la televisión, en los medios de comunicación, en general, y eso puede llevar a información errónea, incorrecta, con mitos, sin mitos y que son necesarios siempre aclararlos con las mujeres (E13M).

\section{En términos similares se pronuncia otra participante del estudio:}

(...) cuando tienen la información necesaria y desde nosotros, desde los centros de salud, desde lo más cercano que tienen, con todo lo que ello implica, (...) hay que evitar el tema de los mitos entonces (...) la autonomía se genera con información, con educación, con consejería (E3M).

La información es vista siempre como un vector de la autonomía y no al revés. La posibilidad de que la entrega de información sea utilizada como una herramienta de coerción no resulta evocada explícitamente. Sin embargo, la tensión emerge a propósito de los eventuales desacuerdos entre la decisión de la paciente y la del personal de salud, algunas veces, revelando la sobreutilización de la consejería como herramienta de presión:

\begin{abstract}
Yo en este caso particular, respeto la opinión de la usuaria y le explico que voy a dejar explícito en su ficha clínica su decisión (...), considerando que puede tener algún, algunos riesgos (...), excepto si, evidentemente, este riesgo (...) es riesgo para su salud vital. Que en caso de riesgo para su salud vital, yo generalmente recurro a algún otro profesional de mi misma línea de manera que refuerce la consejería para ver si existe una forma de abordaje diferente que pueda digamos, a la usuaria permitirle tomar una decisión diferente (E15M).
\end{abstract}

Otras veces, la información constituye una herramienta al servicio de modelos de alta institucionalización del parto (Goberna 2010: 102-103), particularmente presentes en países subdesarrollados, como Chile. El recurso discursivo al papel del feto como destinatario de asistencia, es utilizado en el marco de la entrega de información para vencer las resistencias de las gestantes:
(...) en realidad si nosotros partimos por decir que uno de los pilares fundamentales de la atención de salud de una mujer es la autonomía hay que respetarla, pero también ese respeto va de la mano, dependiendo de uno, de donde esté parado en ese momento, en los diferentes niveles de atención de salud, ya sea en el primario, secundario, terciario, donde muchas veces uno tiene que comenzar a transar con la mujer, en el nivel terciario donde se presentan las urgencias médicas, donde, a pesar de que están los derechos sexuales uno tiene que hacerle ver a la mujer de que está la urgencia de por medio, y que hay un feto de por medio, hay un hijo de por medio y las mujeres lo entienden muy bien, ahora no sé trata de imponerle nuestra visión, sino que informarla (E13M).

Un tercer grupo de limitaciones de la autonomía procreativa se refiere, entonces, a los conflictos materno-fetales. Una de las consecuencias del proceso de medicalización y tecnificación del parto, que viene desarrollándose desde la segunda mitad del siglo XX, es el abandono de una visión de unicidad del embarazo y su reemplazo por una visión dualista. Para esta, el embrión/feto es un paciente, tanto como la gestante, y los intereses de uno y otra se tornan independientes e, inclusive, contradictorios. Así pueden surgir los conflictos maternofetales entendidos como situaciones en que las acciones $u$ omisiones de la gestante pueden provocar daño actual o potencial al embrión o feto (Goberna 2010; Briozzo et al, 2013). A menudo, los y las profesionales de la salud perciben que en estos casos existe una colisión entre el principio de beneficencia - que protege en este caso al feto- y el principio de autonomía.

Cabe mencionar que estos conflictos maternofetales aparecen elaborados por los y las participantes del estudio, la mayor parte de las veces, como ejemplos concretos y no como limitaciones abstractas al principio de autonomía. Sus soluciones son, por consiguiente, variadas. 
En unos casos, el conflicto se resuelve apelando a técnicas jurídicas de control y disciplinamiento, de naturaleza extrahospitalaria:

Cuando hay un embarazo de por medio nosotros podemos recurrir a interponer un recurso de protección para el feto en útero y ese recurso de protección implica hacer un procedimiento legal, el médico dice: 'sabe que yo no la puedo dar de alta, lo siento, yo lo que tengo que hacer es informar', y se hace una, a través de jurídica del hospital, una nota que establece diagnóstico de la paciente, cuáles son los riesgos que ve el médico y se envía a tribunal, tribunal determina a la señora, la señora se va igual tú comprenderás que nosotros no tenemos carabineros para amarrarla y dejarla acostada en la cama, ella se va igual, pero carabineros tiene que ir con la orden del juez - que emite después de este informe - a buscarla eventualmente y traerla de vuelta al hospital, y así las veces que ella se quiera ir tenemos nosotros que informar a tribunales y por supuesto ingresa todo un proceso que también es bien engorroso (E16M).

En otros casos, implica acudir a los Comités de Ética intrahospitalarios:

Hay 2 tipos de comité de ética en el hospital, el comité de ética asistencial y el comité de ética para estudios de investigación ya, cuando se produce ese tipo de dilema (...) cualquiera que sea, tú dices esta mujer se está perjudicando ella, su embarazo o está perjudicando a su hijo por ejemplo, porque no quiere seguir una indicación que tal vez es recomendable uno puede elevar una solicitud al comité de ética para que defina el tema, me entiendes, o sea tú pides ayuda a terceros, a un comité de ética que existe en todos los hospitales (E8G).

Finalmente, el conflicto materno fetal puede resolverse mediante una objeción de conciencia, a condición de que la creencia invocada por las y los profesionales de salud objetores sea, precisamente, el bienestar del feto. Sobre este asunto se volverá en la sección correspondiente.

Gran parte de la literatura contemporánea señala que las discusiones éticas en torno al aborto se vinculan, por un lado, al valor moral de la vida humana prenatal y a los principios de autonomía y dignidad (Dworkin, 1993; Lolas, 1998; Zúñiga, 2018). La posición en contra del aborto afirma que existe una continuidad entre el óvulo fecundado y un ser humano nacido, de suerte que no existe diferencia moral relevante entre ambos estadios de la vida humana, mientras que la defensa liberal del derecho de las mujeres a abortar considera que el nacimientoel hito que determina la existencia legal de una persona- o algún momento posterior al primer trimestre de embarazo, marca una línea divisoria moralmente significativa en lo concerniente a la tutela de la vida humana (Singer, 1995). En consecuencia, las nociones de vida humana y de persona aparecen comúnmente entrelazadas en los discursos en contra o a favor del aborto, operando como correlativos. Es importante precisar que, en las sociedades modernas, la idea de persona equivale a la individuación, es decir, al hecho de ser un sujeto de derecho.

Los resultados del estudio muestran que entre los y las profesionales participantes existen múltiples significados atribuidos a la noción de vida y lo propio ocurre respecto de la idea de persona. No existe una correlación nítida entre los primeros y los segundos.

Para la gran mayoría, la vida es concebida como un acontecimiento social, una biografía, y no sólo como un proceso biológico. Así, por ejemplo, una de las entrevistadas sugiere que: "la vida es el hecho de poder existir, el reaccionar frente a tantas cosas, a lo que te pasa día a día, es toda una historia, entonces es como tan amplia" (E2G). Otra, al preguntarse "¿qué es la vida para mí?", señala:

Es formar parte de algo, formar parte de un todo, de una familia, de un hogar, de una sociedad y poder tú, a través de tu crecimiento y desarrollo poder desarrollarte como persona y poder beneficiarte de eso (E1M). 
Los descriptores de la vida humana, se vinculan, en general, a la conciencia, la voluntad, la búsqueda de un propósito o la independencia. Estos elementos permiten distinguir la vida humana de la existencia de cualquier otro ser viviente:

La vida desde el punto de vista biológico es una serie de procesos bioquímicos que se desarrollan en un ser que es capaz de reproducirse, eso para mí es la vida, es lo más básico, pero la vida humana ya cambia, porque en la vida humana tienes otros aspectos muy fundamentales, no es cierto, que dependen de la capacidad de razonar, de decidir, de aprender, de incorporar experiencias, ideas y desarrollarse desde el punto de vista personal humano, etc., social, o sea es muy distinta la definición de vida, así en seco a vida humana (E7G).

Hay participantes que atribuyen al hecho social/ biológico de vivir una dimensión normativa, es decir identifican la vida, en sí misma, con el derecho de estar vivo: "bueno la vida es el valor y el derecho más inalienable que tenemos, ya, porque a partir de eso somos personas, o sea, es un derecho" (E17M).

Algunas y algunos entrevistados adscriben a una posición en la que no existe diferencia moral significativa entre un organismo biológico de naturaleza humana y una persona: "es que siempre es persona, o sea un organismo biológico humano, por el hecho de ser humano es persona" (E5M).

Sin embargo, la gran mayoría identifica la existencia de una variedad de puntos de vista, tanto desde una perspectiva ético-religiosa como científica. Estos puntos de vista se relacionan siempre con el momento moralmente relevante para transformarse en persona o sujeto de derechos. Por ejemplo, para uno de los entrevistados:

(...) ese es un tema más bien filosófico que científico, hay distintas apreciaciones, desde gente que dice desde el momento de la concepción, pero no es la postura, esa es la postura más bien religiosa, a esta postura más filosófica o científica que dicen que sería una vez que el feto ya tiene conformado su cerebro, que sería alrededor de las 14 semanas, entonces ahí, depende a quién le preguntes o quien investigue (E7G).

La distinción entre persona en potencia (es decir, como posibilidad de ser) y persona en acto (el ser mismo) es evocada reiteradamente, aunque no alude siempre a los mismos hitos. A veces la idea de persona en potencia remite a la fecundación:

(...) biológicamente hablando, yo creo que al momento que los gametos son, de la especie humana, se puede considerar que al menos ese ser biológico es potencial persona potencial persona, pero yo creo que finalmente va a ser persona cuando exprese, no es cierto, desde su potencial digamos ya desarrollado, su forma de ser en el fondo (E8G).

Y, en otros casos, se refiere al nacimiento. Así lo sugiere una entrevistada: "yo creo que cuando nace es una potencial persona" (E9G).

La noción de persona en acto es comprensiva también de un crisol de criterios. Uno de estos criterios es la viabilidad, es decir, la posibilidad de sobrevida que tiene el feto y que plantea un límite, en el discurso de la mayoría de las y los entrevistados, a la eventualidad del aborto en términos generales.

Desde mi punto de vista (...) desde su nivel de desarrollo biológico en el fondo, por lo tanto desde mi punto de vista el desarrollo biológico cerebral o nervioso sobre las 20 semanas, o sea eso para mí es como clave digamos, y por lo tanto la posibilidad que tiene ese ser vivo de desarrollarse como ser humano posterior digamos (E15M).

Otros criterios mencionados son el nivel de desarrollo neuronal como indicador del inicio de funciones humanas complejas, a saber:

(...) para mí cuando todas las conexiones neuronales en sí logran transmitir y aparte de eso interpretar toda la información que arroja el resto de sistemas que están fun- 
cionando. [...] por ejemplo sobre 35 semanas donde ya la transmisión nerviosa de sensaciones en el útero incluso, comienza a conectarse con partes del cerebro que lo racionalizan, hacen sentir sensaciones, emociones, etc. (E11M).

La independencia biológica es un factor que permite definir a la persona, así lo sugiere una de las entrevistadas: "yo creo que cuando puede vivir de manera independiente, cuando no depende al menos biológicamente de otra persona" (E10M).

Esto se complementa con la consideración de la separación material entre feto y gestante, por ejemplo:

Ahora si tú me dices cuándo es persona, es persona cuando ya nació, cuando ya tiene relaciones, antes no es persona, es un embrión, un ente que tiene la potencialidad de desarrollarse, pero persona ya es cuando tiene desarrollo con otros, cuando logra desarrollarse (E6G).

Un último criterio señalado por las y los entrevistados es la conciencia del yo, a saber:

\begin{abstract}
Según yo lo consideran persona desde el momento en que nace, pero yo creo (...) puede ser desde el momento en que tenga un poquito más de conciencia, tú mucho no te acuerdas cuando eres un bebé, entonces cuando tú empiezas a tener recuerdos, a tener vivencias o a tener más opinión puedes desenvolverte más como persona, pero no sé por ahí (E18M).
\end{abstract}

Cabe mencionar que la persona no solo es el resultado de la reunión de ciertos requisitos intrínsecos, también puede ser el producto de un acto social de singularización de carácter simbólico (es decir, por la palabra). En esta concepción, la individuación se produce cuando un ser humano es dotado de un nombre, una identidad registral $\mathrm{o}$, inclusive, mediante el bautizo:

desde que está en el útero, es una personita que vive, que siente, que escucha, responde a lo que tú le dices, que tiene nombre, las mamás le ponen nombre, que generan un vínculo, forma parte de algo (E1M).
Es de destacar que, de acuerdo con esta perspectiva, la singularidad no proviene de una cualidad inherente al ser humano (en este caso del embrión/feto) sino que es transmitida por otro, cuya singularidad, a su vez, no está en duda (Boltanski 2004: 78).

\section{Objeción de conciencia: concepto, fundamento y estructura}

La discusión acerca de la objeción de conciencia en Chile ha estado asociada principalmente a los temas de salud reproductiva (Figueroa 2016, Montero, 2014). Esta discusión tomó fuerza a partir de la presentación del proyecto de despenalización de la interrupción del embarazo en tres causales (21 de mayo 2014), se renovó con motivo de la impugnación ante el Tribunal Constitucional del referido proyecto y se ha extendido a lo largo del accidentado intento de establecer una regulación administrativa para la objeción de conciencia. En efecto, el gobierno saliente de Michelle Bachelet dictó un primer protocolo sobre objeción de conciencia ${ }^{3}$, que fue reemplazado por otro protocolo dictado por el gobierno de Sebastián Piñera. ${ }^{4}$ Este último fue declarado ilegal por la Contraloría General de la República ${ }^{5}$. Finalmente, se dictó el Reglamento $N^{\circ} 67$ para ejercer objeción de conciencia según lo dispuesto en el art. 119 ter del Código Sanitario (en adelante; ROC).

Desde el punto de vista de la política de atención de salud, el debate sobre la objeción de conciencia ha tenido, como eje principal, su alcance y su relación con los principios bioéticos que dirigen la práctica médica. En las siguientes

Resolución Exenta № 61, de 22 de enero de 2018

4 Resolución Exenta N ${ }^{\circ}$ 432, de 22 de marzo de 2018

5 Dictamen 011781 N18, de 09 de mayo de 2018 
líneas se abordará esta cuestión, con base en los discursos recopilados en este estudio, los que fueron recogidos con anterioridad a la vigencia de la ley, sus protocolos intermedios y del ROC antes mencionado.

\section{Concepto de objeción de conciencia}

A pesar de que al momento del estudio la objeción de conciencia no se encontraba regulada en el ordenamiento jurídico chileno, las y los participantes del estudio hacen referencia a una serie de acciones en la práctica médica que remiten a casos en los que un procedimiento de salud reproductiva podría no ser suministrado por ser incompatible con las creencias de los o las profesionales.

Jurídicamente la objeción de conciencia puede entenderse como "aquella figura que permite al individuo negarse o rehusarse a cumplir una obligación jurídica, cuando la actividad correspondiente signifique la realización de conductas que pugnan con sus convicciones íntimas" (Pardo 2006: 56).

Los discursos recogidos dan cuenta de que la mayoría de las y los entrevistados - a excepción de 3 profesionales - tienen conocimiento sobre la objeción de conciencia. Sin embargo, sus opiniones muestran una diversidad de aproximaciones a su ejercicio.

El concepto de objeción de conciencia, en general, está asociado a un acto que busca resguardar la integridad o menoscabo moral, por ejemplo:

(...) es como, cuando uno tiene impedimentos morales, no es morales, no sé si es morales la palabra, o éticos, que no van de acuerdo con sus creencias o deja de hacer algo, o evita hacer algo por lo mismo (E1M).
Es considerado un derecho humano, asociado a la libertad de pensamiento y religión, y que sustenta el derecho a la inacción. De esta manera, uno de las informantes refiere que la objeción de conciencia:

(...) es como la libertad que yo tengo de no actuar, principalmente en un procedimiento, cuando ese procedimiento va en contra de mis principios éticos, éticos y/o morales, principalmente por ahí (E5M).

Asimismo, otra entrevistada plantea:

Lo que podría entender, como en simples palabras, es el no realizar una atención o no prestar una atención por mis creencias personales, como te decía al principio, sean religiosas, sociales, qué se yo. (E14M).

Un aspecto importante para considerar es que la negación a realizar un determinado procedimiento médico tiene relación con no obedecer un mandato normativo considerado injusto, en ese sentido, como lo plantea Rawls (Cit. en Figueroa 2016: 154) "la objeción de conciencia es una forma de desobediencia al derecho que consiste en oponerse a cumplir un mandato legislativo o una orden administrativa". Así se expresa en la siguiente cita: "bueno la objeción de conciencia es en el fondo negarse a una normativa, algo que impone una autoridad, no sé, por un tema ético, moral, religioso" (E15M).

En varias opiniones se observa que el proceso de objeción de conciencia es unipersonal y autónomo, es decir, que compete sólo la decisión del o la profesional involucrada. Sin embargo -en algunas entrevistas- se da cuenta que esa autonomía tiene ciertos límites asociados fundamentalmente al rol profesional entendido como de servicio y a los principios bioéticos que lo ordenan, especialmente de beneficencia y no maleficencia. Por ejemplo: 
Yo creo que en ese sentido uno tiene que separar lo que es su propio pensamiento de lo que es el ejercicio profesional, uno no está aquí para influir de acuerdo a sus pensamientos, sino que está en una labor de servicio público y de servicio al prójimo, si no es servicio público servicio al prójimo y no para imponer sus propias creencias, por lo tanto creo que yo los valores personales - las creencias personales están para tú quehacer personal y, por lo mismo, es que yo pienso que la objeción de conciencia tiene que ser un tema bastante restringido no puede ser muy generalizado (E7G).

Solo una de las y los entrevistados sugiere que la objeción de conciencia debe contemplar un proceso de reflexión personal que permita definir las razones para objetar, a saber:

(...) para mí la objeción de conciencia implica que uno debería hacer una revisión interna y ver en realidad por qué objeto, por qué no estoy de acuerdo (...) la objeción de conciencia es porque mi conciencia dice que esto no es lo correcto y está basado en valores, en historias de vida de cada uno y en la vida que uno lleva en el fondo (E16M).

Aunque el ROC no condicione el ejercicio de la objeción de conciencia a la justicia de las razones invocadas por quien desea incumplir la obligación legal o administrativa, en la literatura jurídico-filosófica la legitimidad de esas razones constituye un debate central (Figueroa 2016, 155; Alegre 2010).

Por otra parte, para la gran mayoría de las y los participantes del estudio la objeción de conciencia expresa un conflicto entre el derecho del o la profesional a objetar y el derecho del o la paciente a decidir. Por ejemplo, una entrevistada señala:

(...) porque cuando estamos hablando (...) de hecho las 3 causales del aborto, de la despenalización del aborto ni siquiera tiene un poco que ver, siento yo, con que la persona diga 'yo no quiero tener este hijo' (...) y si siento que la persona que está al otro lado, que es el profesional, como yo creo en la auto gobernanza digamos y en el ejercicio de los derechos y el respeto a los derechos de las personas, los profesionales también (...) son sujetos a eso, de que se les respeten sus derechos digamos (E17M).

No obstante, el derecho del objetor encuentra límites en los principios éticos de beneficencia y de no maleficencia, los que configuran la prestación de salud como un acto de servicio. En los discursos examinados, el primero de estos principios se asocia implícitamente al servicio de Otro, quien debe ser informado y educado para el ejercicio de sus derechos. El principio de no maleficencia, es decir, evitar y reducir al mínimo el riesgo y daño para el afectado (Lolas y Outomuro 2006) genera, a su turno, la obligación moral de evitar las repercusiones negativas que la objeción de conciencia acarrea para las y los pacientes. Sin embargo, estos principios, que debieran considerar especialmente la opinión de estos últimos, en la práctica sólo toman en cuenta la perspectiva del o la profesional, quien tiene la autoridad otorgada por su saber.

Para las y los profesionales entrevistados, el principio de no maleficencia se expresa de forma diferencial en los distintos niveles del sistema de salud en el que se desempeña el o la profesional, a saber, primario, secundario y terciario ${ }^{6}$. Esto es consistente con lo dispuesto en el Protocolo sobre Acompañamiento y Atención Integral a la Mujer que se encuentra en alguna de las tres causales que regula la ley 21.030 (2018). En este reglamento-que no regula la objeción de conciencia- se distinguen procedimientos

\footnotetext{
La organización del sistema de atención en salud en Chile distingue tres niveles: Atención Primaria de Salud, que representa el primer nivel de contacto de las y los individuos, la familia y la comunidad con el sistema público de salud, brindando atención ambulatoria; Secundaria, que corresponde a atenciones y procedimientos de especialidades ambulatorias, alimentados de solicitudes de interconsultas generada principalmente de Atención Primaria y de otras especialidades y Terciaria que comprende la atención de urgencia hospitalaria y la atención cerrada de Hospitalización, en el cual son incluidas las intervenciones quirúrgicas complejas.
} 
y acciones vinculados a la interrupción del embarazo, asociados a cada uno de esos tres niveles de intervención.

En el caso de nivel primario, se plantea, como condición para formular objeción de conciencia, la existencia de otros recursos profesionales que aseguren la derivación oportuna. Esto se expresa principalmente en el discurso de matronas y matrones que se desempeñan en Atención Primaria de Salud (APS), a saber:

\begin{abstract}
Yo derivo e informo a las pacientes también, no es que las derive porque si nomás, en general todas entienden. A veces yo tengo a mi otra colega al frente, le digo, hablo con ella y si me dice 'mándala para acá' y la mando altiro, y ellas igual entienden eso, en general es así como rápido. Ahora si estoy sola, justo sola, sola y no hay ninguna otra colega, le tengo que decir que venga otro día, pero yo hago todo el trámite, yo me contacto con mi colega, le pido la hora, me aseguro que ella la vaya a ver, no la dejo en el aire, así como ya vaya y pida hora con otra colega, no (E1M).
\end{abstract}

En el caso del nivel terciario, es decir, centros de alta complejidad, la derivación es más difícil, pues la disponibilidad de recursos profesionales es más escasa, debido a la alta especialización. En esta situación, la objeción de conciencia se tensiona con el principio bioético de no maleficencia en los casos de riesgo de morbilidad o mortalidad del paciente.

(...) si estoy en un lugar aislado donde soy la única persona que puede hacer el procedimiento, puede acompañar este procedimiento, ese derecho de objeción de conciencia no puede estar sobre el derecho de la otra persona, o sea tendría que en ese caso extremo saltarme la objeción de conciencia, porque tampoco es absoluta, también tienen que haber ciertos matices, respecto a cuándo y lamentablemente no voy a poder optar por esta objeción, porque (...) soy la única persona que puede estar en ese procedimiento, no hay otros profesionales de salud, la paciente, no sé, está en peligro de muerte, o sea no puede ser absoluto, en ese caso claro que no, pero mientras esté la opción de que alguien me reemplace, de que exista alguien que no tenga la misma objeción, yo no creo que debiera haber ningún problema (E12M).
En algunos casos, se desprende una relación implícita entre la objeción de conciencia y el principio bioético de la justicia, entendido como la distribución ecuánime de los recursos, derechos y obligaciones y que debe ser respetado por cada una de las partes (Lolas y Outomuro 2006). Con todo, esta relación no se expresa como un derecho de las y los pacientes sino como una obligación derivada del carácter estatal de la prestación de salud pública y, por tanto, de su obligatoriedad. Así, por ejemplo, se expresa:

(...) por otro lado, sobre todo el sistema público no puede tener objeción de conciencia porque se supone que (...) es una entidad que representa al Estado y si el Estado te está diciendo que salió esta ley [que regula la despenalización de la interrupción voluntaria del embarazo en tres causales], que tú tienes que acatar, como institución no puede negarse, o sea, eso sí que está prohibido, como institución (E12M).

Otra entrevistada, respecto a ese mismo punto señala:

(...) la objeción de conciencia como normativa en el hospital no existe, y no existe porque en el fondo nosotros deberíamos subentender que si trabajamos en un servicio público es para atender todo lo que llegue (...) Desde el punto de vista de la salud pública me parece un poco complejo porque nosotros, nuestro estatuto administrativo, normativa nuevamente, establece que nosotros tenemos que atender en igualdad de condiciones, independiente del credo religioso, de cualquier diferencia, entonces yo creo, si me preguntas así como a mí no me había cuestionado eso la verdad porque no me daba paso para ello, yo creo que si uno es funcionario público tiene que atender lo que llega y si hay un valor que me dice a mí que va tan reñido con la institución creo que mi derecho es, para hacer valer mi propia autonomía al respecto, es alejarme de esa institución, pero es un tema mío no dejar de atender a alguien porque esa institución está para atender a esa gente $(\mathrm{E} 16 \mathrm{M})$. 


\section{Objeción de conciencia y derechos reproductivos}

En cuanto a la objeción de conciencia y el ejercicio de los derechos reproductivos, especialmente de las mujeres, en los discursos se observa un sesgo respecto de la autonomía reproductiva femenina. Las decisiones sobre control de la natalidad, especialmente la esterilización, el dispositivo intrauterino (DIU) y el aborto; son las más referidas por las y los consultados.

Por ejemplo, en relación con la decisión de esterilización una entrevistada plantea:

\begin{abstract}
Ahora no, ahora la paciente solicita su esterilización y no hay problema en que se realice, pero generalmente igual se cuestiona de cuántos hijos ha tenido, si es muy joven o no, si se puede arrepentir o no, pero antiguamente era peor, porque antiguamente se le pedía [pausa] si la pareja no estaba de acuerdo no se esterilizaba [pausa]. Está totalmente limitado el derecho reproductivo cuando hablamos de esterilización porque finalmente (...) te cuestionan eso, te cuestionan la edad, si has tenido o no hijos, no es quizás se va arrepentir, pero puchas si se arrepiente es cosa de ella, por eso te digo la salud está acostumbrada a decidir por las personas y puchas si se arrepiente se tratará de recanalizar las trompas y si no le resulta, no le resulta, pero, si yo creo que ahí no se respeta mucho, sobre todo cuando son mujeres que no han tenido hijos, que son jóvenes, no sé, le ponen todas las trabas para que no lo realice $(\mathrm{E} 12 \mathrm{M})$.
\end{abstract}

En lo que concierne al DIU, la objeción de conciencia se vincula al supuesto de que éste, al impedir la anidación del ovulo fecundado, "podría afectar el proceso de una vida" (E15M). Una de las entrevistadas plantea:

(...) lo que sí yo he actuado en una cosa con objeción de conciencia en lo que es cuando uno pone el dispositivo intrauterino, todas mis colegas saben y mis pacientes saben que yo no pongo DIU porque dentro de los riesgos que hay está el riesgo de embarazo y ese embarazo puede terminar con aborto, pero yo explícitamente lo digo, o sea si alguien viene a pedirme que le ponga un DIU, yo le cuento todas las ventajas, desventajas, mecanismo de acción, pero le digo que la voy a derivar a otro profesional porque yo no lo hago por tales motivos y la gente no tiene ningún problema, nunca les digo 'oh está mal lo que está haciendo, cómo se le ocurre' no, no le digo eso, le digo lo que yo pienso y por qué lo hago, no por eso tampoco se lo niego (E1M).

Algunas de las opiniones que defienden la objeción de conciencia en caso de aborto, invocan las dificultades de los procedimientos médicos, especialmente de aquellos asociados a la interrupción del embarazo luego de las diez semanas:

Yo creo que aquí hay un tema que no se ha discutido y es la dificultad técnica de practicar un aborto en un embarazo avanzado. Eso tiene complicaciones, no todos los médicos están en condiciones de hacerlo, ni aun los ginecólogos, es algo que uno supone que lo van a hacer, pero no es tan así, es una técnica que tiene sus dificultades, no es una técnica sencilla ¿y quién se hace cargo de las complicaciones? (...) y resulta que después tienes un resultado desastroso con la madre y entonces ¿quién responde por eso? (...) en general, sobre las 10 semanas ya es complicado, entonces, y tenemos un límite de 14 semanas, entre las 11 y 14 semanas no es para cualquier ginecólogo, ni siquiera para cualquier ginecólogo y sobre las 14 y las 18 es un tema que aquí, por ejemplo aquí que hay mucho ginecólogo no creo que haya más allá de 2 o 3 que lo puedan hacer, es un tema complejo, muy complejo (E7G).

Sin embargo, se puede observar que la justificación técnica solapa una preocupación por las implicancias jurídicas relativas a la responsabilidad médica, de un riesgo de daño, a saber:

(...) entonces de repente, a veces hay urgencia en parto en que hay que 'pucha llamen a la anestesista porque hay que hacer un fórceps de urgencia porque hay un sufrimiento fetal' y la anestesista no puede venir, tenemos que poner nosotros anestesia, a veces, ya, y mientras no pase nada todos te dicen '¡oh qué bueno!' te aplauden la decisión 'qqué buena tu decisión, qué regio!', pero si te pasó un accidente anestésico te van a colgar, los mismos que te aplaudieron te van a colgar y te van a entregar en bandeja para que el juez te haga papilla en el juzgado (E6G). 
Por otra parte, las entrevistas sugieren que el alcance de la objeción de conciencia depende de las causas de la interrupción del embarazo. En el caso del aborto por inviabilidad fetal, en general, el principio de no maleficencia actúa en favor de la gestante. Por ejemplo, una de las entrevistadas expresa:

\begin{abstract}
Yo antes de eso también tenía objeción de conciencia, pero cuando llegó la señora, es imposible tenerla, no puedes ser tan mala y dejar a esa pobre mujer con una guagüita que tú sabes, que lo más seguro es que en un par de días hubiese fallecido sola en el útero, porque no venía muy bien. Entonces como que ahí te replanteas todo de nuevo, todas tus creencias, toda tu ideología y te das cuenta que en verdad tú no tienes ni un derecho a decidir por sobre otra persona que quiera hacer lo que ella quiera hacer con su vida, porque por algo lo está haciendo si no es porque le dio la gana (...) (E18M).
\end{abstract}

Correlativamente, el alcance de la autonomía reproductiva de la gestante está también delimitada por las causas que determinan el aborto. En varias entrevistas se observa la distinción entre el aborto por razones médicas y el aborto decidido libremente.

En relación con este último, las opiniones están divididas. Aquellas y aquellos que no están en desacuerdo con respecto al aborto amplio, defienden el respeto de la autonomía de las pacientes. Por ejemplo, una entrevistada señala:

Mi opinión personal respecto de la interrupción del embarazo (...) yo creo que toda mujer debiese tener la posibilidad de decidir si quiere o no, primero (...) súper clara si quiere o no tener un hijo y después si llega a embarazarse debería tener la posibilidad de elegir si quiere o no continuar con ese embarazo (E15M).

Especialmente en el caso de ginecólogas y ginecólogos, se constata una perspectiva más pragmática respecto del aborto libremente decidido. Por ejemplo, uno de ellos sugiere:
A ver, yo estoy personalmente en contra del aborto voluntario, sin embargo estoy a favor de una ley que ampare el aborto voluntario, no sé si se entiende [ríe] es como que quiero estar bien con Dios y con el Diablo, lo digo porque, porque en el fondo el aborto voluntario existe, ya, entonces hoy en día el aborto voluntario existe, de alguna manera existe, sin control, sin ninguna regulación, ya, y creo que a veces podría ser peligroso, me entiendes, creo que es mejor que alguien se haga responsable de eso y la mujer también se haga responsable en un tema que puede afectar su salud, me entiendes, a que se haga, digamos, de forma clandestina o poco transparente ya, a pesar de que no soy partidario del aborto me entiendes, pero el mal menor sería que mejor fuera una cuestión regulada $(E 8 G)$.

También se plantea la necesidad de que el aborto libremente decidido esté regulado en lo relativos a los plazos, considerando las dificultades técnicas del procedimiento en una etapa muy avanzada. Una de las entrevistadas menciona:

(...) no estoy en desacuerdo, pero sí con límite gestacional porque es muy complejo a mayor edad gestacional [pausa] o sea ojalá lo antes posible, porque después es muy complejo, ya de la semana 12, 14 en adelante es cada vez más terrible, con riesgos para la madre, muchas complicaciones, mucha dificultad y mientras más precoz mejor (E9G).

Quienes manifiestan su desacuerdo con el aborto libremente decidido sostienen, como fundamento, el valor de la vida y sus creencias personales. Por ejemplo, una entrevistada señala:

(...) personal, no estoy de acuerdo, pero por una creencia cristiana, personal, profesional, en realidad, como decía antes yo le informo a la paciente los riesgos, las ventajas o los beneficios que pueda tener y que ellas decidan, no les hago tampoco saber lo que yo opino acerca de (...) (E1M).

\section{Otra entrevistada explica:}

Yo soy católica y soy cristiana y considero que no tenemos el derecho de decidir quién vive o quién no vive en nuestras manos, entonces yo no practico abortos, yo 
puedo realizar todo lo que son las labores que manden sobre la regulación de la fecundidad, pero yo soy contraria al aborto y yo no lo practicaría ni a mí, ni a nadie (E17M).

Asimismo, se observa en ciertas intervenciones una tendencia a evitar caracterizar la interrupción del embarazo avanzado (más allá de las veinte semanas) como un aborto definiéndolo como "un parto prematuro inmaduro causado por el riesgo vital que tiene la madre":

(...) distinto es las 3 causales (...) si yo tengo una paciente, por ejemplo, que tiene una preclamsia severa, un cuadro de hipertensión severo, una insuficiencia renal que producto del embarazo está condicionando su vida yo lo único que puedo hacer es llegar a las 27, 28 semanas y suspender ese embarazo, porque si la paciente se está muriendo [pausa] hay muchos embarazos que nosotros los interrumpimos a las 27, 28 semanas por la gravedad materna (...) esa guagua de 27, 28 semanas pesa 900 gramos, puede tener chance de vivir, pero si no lo saco se van a morir los 2, ya, entonces, por ejemplo, hay condiciones en que me obliga a eso parto prematuro inmaduro causado por el riesgo vital que tiene la madre (...) la mentalidad no es que yo estoy condicionando un aborto, entonces si se hace interrupción de embarazo precoz, pero depende del marco legal en el que tú lo pongas como lo respaldes" (E6G).

Esta sustitución de categorías permite diluir el conflicto ético en relación a la interrupción del embarazo, sustentándola en la norma técnica médica, a saber, la "pérdida del producto de la concepción menor a 20 semanas y/o menor a 500 gramos" (E6G). Esto difiere de las reglas jurídico-penales que regulan el aborto en Chile, las que no atienden al momento en que se produce la interrupción del embarazo sino al propósito subjetivo del agente, es decir, buscan verificar si la interrupción del embarazo es maliciosa o no.

\section{Conclusiones}

El análisis de los discursos de las y los profesionales de salud sexual y reproductiva de la ciudad de Valdivia permite concluir que los avances normativos respecto de la ciudadanía biológica se ven limitados por el saber médico y las convicciones morales de estos profesionales.

La concepción de autonomía dominante en la práctica clínica es débil. La autonomía no es concebida como un atributo intrínseco del o la paciente, sino que resulta moldeada por el conocimiento y convicciones morales de las y los profesionales. En algunos casos, el ejercicio de la autonomía está sujeto a la información proporcionada por las y los especialistas y, en otros casos, está delimitado por las convicciones morales de cada profesional. Se trata, entonces, de un modelo de autonomía tutelada que opera a través de diversas estrategias. Algunas de estas son de naturaleza institucional, como la consejería, la educación o la intervención de los Comités de Ética. Otras son de carácter individual o personal, como la objeción de conciencia. Esto corrobora la existencia de un modelo de salud altamente centrado en el saber científico y de una configuración de la relación clínica con un sesgo individualista.

Los discursos examinados dan cuenta de que las limitaciones a la autonomía son, a menudo, justificadas apelando al respeto de los principios bioéticos que regulan la práctica clínica y que remiten al bienestar de las y los pacientes (beneficencia y no maleficencia). La objeción de conciencia hace excepción a lo anterior. Esta última se estructura, más bien, como un conflicto nítido entre las concepciones de las y los pacientes y las concepciones de cada profesional, siendo estas últimas prevalentes. 
No se requiere que estas concepciones se identifiquen con el bienestar de ningún paciente.

La objeción de conciencia se relaciona siempre con concepciones sobre la vida humana, las que tienen un carácter abstracto. Las concepciones de la vida de las y los profesionales de la salud entrevistados no necesariamente son de naturaleza científica, en muchos casos responden a concepciones religiosas.

El conflicto moral que encierra la objeción de conciencia se plantea como una disputa entre dos individuos, a saber, el o la profesional de la salud y el o la paciente. Esta configuración oscurece las repercusiones colectivas de la objeción de conciencia lo que se evidencia en la omisión generalizada de reflexiones en torno al principio bioético de la justicia y, en particular, en torno a la igualdad de género respecto de la atención de salud. Los discursos no evocan ningún planteamiento crítico respecto de las consecuencias que un uso excesivamente liberal de la objeción de conciencia puede tener en el ejercicio de la autonomía reproductiva por parte de las mujeres. Al contrario, varios de los discursos analizados otorgan, de entrada, mayor valor a la vida prenatal que a la capacidad de decisión de la gestante en lo relativo a su propia salud. Esto sugiere la permanencia de un modelo biomédico androcéntrico en la atención de salud reproductiva que no descansa únicamente en la autoridad científica de las razones que justifican las prácticas del personal de salud sino, preponderantemente, en una relación jerárquica de poder entre especialista y paciente.

\section{Bibliografía}

Alegre, M. 2010. "Opresión de conciencia. La objeción de conciencia en la esfera de la salud sexual y reproductiva". Derecho y sexualidades. SELA. Buenos Aires: Libraria.12-36.

Boltanski, L. 2004. La Condition fotale. Une sociologie de l'engendrement et de l'avortement. París: Gallimard

Boyer, A. 2012. "Biopolítica y filosofía feminista". Revista de Estudios Sociales 43, 131-138.

Briozzo L., Nozar, F., Coppola, F. y Fiol, V. 2013. "Abordaje clínico del conflicto de interés materno fetal y sus relación con el estatus del feto como paciente". Revista Médica del Uruguay 29 (3):187-194.

Canales, M. 2006. Metodologías de la investigación social. Santiago: LOM Ediciones

Casas, L. y Dides, C, 2007. "Objeción de conciencia y salud reproductiva en Chile. Dos casos paradigmáticos”. Acta Bioethica 13 (2): 199-206.

Dworkin, R. 1993. El dominio de la vida: una discusión sobre el aborto, la eutanasia y la liberal individual. Barcelona: Ariel.

Figueroa, R. 2016. "Objeción de conciencia y aborto". Debates y reflexiones en torno a la despenalización del aborto en Chile, Casas, L. y Laeson, D. (Comps.). Santiago de Chile: LOM.147-178.

Foucault, M. 2010. Nacimiento de la biopolítica. Curso en el Collège de France (1978-1979). Buenos Aires: Fondo de Cultura Económica
Foucault, M. 2003. The Birth of the Clinic. London: Routledge.

Foucault, M. 1993. Historia de la sexualidad. La voluntad de saber. Madrid: Siglo XXI.

Goberna, J. 2010. "Mujeres y procreación: El respeto a la autonomía en la relación asistencial". ¿Qué dignidad? Filosofía, Derecho y práctica sanitaria? Boladeras, M. (Ed.). Proteus: Barcelona, 105-157.

Kippendorff, K. 1990. Metodología del análisis de contenido. Teoría y práctica. Buenos Aires: Ediciones Paidós.

Lemm, V. (ed.). 2010. Michel Foucault: neoliberalismo y biopolítica. Santiago, Ediciones Universidad Diego Portales. 2010.

Ley $N^{\circ} 20.584$ que regula los derechos y deberes que tienen las personas en relación con acciones vinculadas a su atención en salud. Diario Oficial de la República de Chile, Santiago, Chile. 24 abril 2012.

Lolas, F. y Outomuro, D. 2006. "Bioética y salud pública". Investigación en salud Dimensión ética. Lolas, F., Quezada, A., y Rodríguez, E. (Eds.). Chile: Andros, 103-115.

Lolas, F. 1998. Bioética. El diálogo moral en las ciencias de la vida. Santiago: Editorial Universitaria.

Llober-Valls, C. 2009. Mujer, Salud y Poder. Madrid: Cátedra

Miles, 2016. Salud sexual, salud reproductiva y derechos humanos en Chile. Dides, C. y Fernández, C., (Eds.). Chile: Miles. Ministerio de Salud, 2018. Norma general técnica $n^{\circ} 197$ 
sobre acompañamiento y atención integral a la mujer que solicita la interrupción voluntaria del embarazo en tres causales. Núm. 129 exenta.- Santiago, 2 de febrero de 2018.

Ministerio de Salud, 2018. Protocolo Para la Manifestación de Objeción de Conciencia Personal y para la Objeción de Conciencia Invocada por Instituciones en el Marco de lo Dispuesto en el Artículo 119 ter del Código Sanitario. Santiago, 27 de enero de 2018

Ministerio de Salud, 2018. Protocolo para la manifestación de objeción de conciencia según lo dispuesto en el artículo 119 ter del Código Sanitario y deja sin efecto resolución exenta $\mathrm{N}^{\circ} 61$ de 22 de enero de 2018. Santiago. 22 de marzo de 2018.

Ministerio de Salud, 2018. Reglamento para ejercer objeción de conciencia según lo dispuesto en el artículo 119 ter del código Sanitario. Reglamento $N^{\circ} 67$ de 29 de junio de 2018. Santiago, 23 de octubre de 2018.

Montero, A. 2014. "La objeción de conciencia de los profesionales y su relación con la atención en salud sexual y reproductiva de adolescentes en Santiago de Chile". Acta Bioethica 20 (2): 197-206

Novas, C. y Rose, N. 2003. "Biological Citizenship". Global Asemblages. Technology, Politics and Ethics as Antropological Problems. Ong, A. y Collier. S. (eds.) USA: Blackwell, (2003):
439-463.

Pardo, C. (2006). La objeción de conciencia en la jurisprudencia de la Corte Constitucional Colombiana. Revista Persona y Bioética 10 (1), 52-68.

Petchesky, R. 2009. "Políticas de derechos sexuales a través de países y culturas: Marcos conceptuales y campos minados". Políticas sobre sexualidad. Reportes desde las líneas del frente. Parker, R., Petchesky, R. y Sember, R. (eds). Perú: Sexuality Policy Watch.

Solitario, R., Garbus, P. y Stolker, A. 2008. Derechos, ciudadanía y participación en salud: su relación con la accesibilidad simbólica de los servicios. Anuario de Investigaciones 15:263-269

Stake, R. 2005. Investigación con estudios de casos. Madrid; Ediciones Morata SRL.

Singer P. 1995. Ética práctica. Londres. Cambridge University Press.

Vallés, M. 2009. Entrevistas Cualitativas. Madrid: Centro de Investigaciones Sociológicas.

Vasilachis, I. 2006. Estrategias de investigación cualitativa. Madrid: Editorial Gedisa. S.A.

Zúñiga, Y. 2018 "Los debates sobre el aborto. Paradojas, narrativas y dispositivos de poder-saber". Anales de la Cátedra Francisco Suárez 52:91-111. 